THE aim of the present study was to verify whether a single oral dose of methylprednisolone could modulate the exercise-induced release of polymorphonuclear neutrophil (PMN) elastase and myeloperoxidase. Four healthy, male subjects were submitted to a $20 \mathrm{~min}$ downhill run $(-20 \%)$ at $60 \% \mathrm{VO}_{2} \max , 3 \mathrm{~h}$ after oral absorption of a placebo or a single dose of $32 \mathrm{mg}$ methylprednisolone. A marked neutrophilia $(+103 \%$ of basal PMN count; $p<0.02)$ was observed $3 \mathrm{~h}$ after methylprednisolone ingestion. During both exercise trials, placebo and methylprednisolone, PMN counts were increased by $46 \%$ and $19 \%(p<0.05)$, respectively. The running test caused marked and significant $(p<0.05)$ increases in plasma myeloperoxidase concentration (MPO). The magnitude of MPO changes was the same in the two trials $(+110 \%)$. Exercise also resulted in significant changes in plasma elastase concentration (EL) in both experimental conditions (placebo: $+104 \%, p<0.05$; methylprednisolone: $+338 \%, p<0.005$ ). Plasma elastase levels reached at the end of exercise on methylprednisolone were significantly higher than after placebo $(p<0.05)$. A significant relationship was found between EL and PMN in methylprednisolone trial only $(r=0.72 ; p<0.005)$. These results showed that the transient exercise-induced release of elastase and myeloperoxidase were not decreased by methylprednisolone.

Key words: Eccentric exercise, Elastase, Methylprednisolone, Myeloperoxidase, Neutrophil, Polymorphonuclear

\section{Effects of methylprednisolone on exercise-induced increases of plasma levels of polymorphonuclear elastase and myeloperoxidase in man. Preliminary results}

\author{
G. Camus, ${ }^{1,2, C A}$ J. Pincemail, ${ }^{2}$ \\ G. Deby-Dupont, ${ }^{2,4}$ C. Deby, ${ }^{2}$ \\ A. Juchmès-Ferir ${ }^{3}$ and M. Lamy ${ }^{4}$
}

${ }^{1}$ Laboratory of Human Applied Physiology, ISEPK, B21, ${ }^{2}$ Center for the Biochemistry of Oxygen, Institute of Chemistry, B6, ${ }^{3}$ Department of Clinical Biology, ${ }^{4}$ Department of Anesthesiology, CHU, B35 University of Liège, Sart Tilman, 4000 Liège, Belgium

${ }^{\mathrm{CA}}$ Corresponding Author

\section{Introduction}

The occurrence of transient exercise-induced polymorphonuclear neutrophil (PMN) activation in man has been demonstrated by several studies based on the measurement of the plasma concentration of specific markers of PMN activity. ${ }^{1-5}$ Increased plasma levels of elastase (EL) $)^{1,5}$ and myeloperoxidase (MPO) ${ }^{2,4,5}$ have been reported in subjects submitted to various exercise protocols. It has been suggested that PMN activation could be a part of an acute inflammatory response to muscle and/or adjacent connective tissue damage. ${ }^{3,5}$ This working hypothesis was substantiated by recent studies arguing for the involvement of complement activation $^{4}$ and damaging contractions in the process of PMN activation. ${ }^{5}$ To date, there are few data concerning the involvement of other mediators capable of triggering or modulating the PMN activity during exercise. Among these mediators are several lipoxygenase and cyclooxygenase products of arachidonic acid (AA) metabolism, such as prostaglandins and leukotrienes. As inflammatory mediators derived from arachidonic acid (AA) were found to be released during exercise, ${ }^{6}$ it seems reasonable to suggest that they could be involved in the inflammatory response to damaging contractions, and therefore, in the exercise-induced PMN activation. Consistent with this view are the results of a recent study by Hasson $e t a l^{7}$ where a potent anti-inflammatory agent (ibuprofen) decreased the delayed onset muscle soreness (DOMS), a symptom of acute inflammation, ${ }^{8}$ in subjects submitted to strenuous exercise. As glucocorticoids are thought to exert their anti-inflammatory effects by inducing the synthesis of lipocortins which, in turn, inhibit phospholipase $A_{2}$ production of arachidonic acid, ${ }^{9}$ we investigated whether methylprednisolone could influence the transient exercise-induced increase of plasma elastase and myeloperoxidase concentrations. It has been shown that the easiest way of producing muscle injury - and therefore, an inflammatory response to exercise - is to force the muscles to contract while lengthening, ${ }^{8,10}$ as in running downhill. These findings prompted us to study the effects of a single oral dose of methylprednisolone on the exercise-induced changes in plasma levels of elastase and myeloperoxidase in subjects submitted to a 20 min downhill run on an inclined treadmill.

\section{Methods}

Four healthy male subjects (mean age $=22 \pm 2$ years; mean body weight: $74 \pm 2 \mathrm{~kg}$ ) volunteered to take part in the present investigation after 
approval of the experimental protocol by the Ethics Committee. Informed consent was obtained from each subject after the nature of the study had been fully explained. During the study period, the subjects were asked to abstain from taking any form of medication. The subjects' maximal oxygen uptake $\left(\dot{\mathrm{VO}}_{2} \mathrm{max}\right)$ was measured during the course of a graded exercise test to volitional exhaustion on a $10 \%$ inclined treadmill as described previously. ${ }^{11}$ Oxygen uptake $\left(\mathrm{VO}_{2}\right)$ was measured by an open circuit method (Ergo-Oxyscreen, Jaeger). The running speeds corresponding to steady state $\dot{\mathrm{VO}}_{2}$ equal to $60 \%$ of individual $\dot{\mathrm{VO}}_{2}$ max were calculated using the nomogram of Margaria et al. ${ }^{12}$ Two weeks after $\dot{\mathrm{VO}}_{2} \max$ measurement, the subjects reported to the laboratory to complete the first of two $20 \mathrm{~min}$ downhill runs on a $20 \%$ grade. The second run was separated from the first by a period of at least 3 weeks during which the subjects were asked to refrain from strenuous exercise. These two trials were performed in the morning after an overnight fast following the same protocol.

A teflon catheter was inserted into an antecubital vein and sealed. Four blood samples of $10 \mathrm{ml}$ were collected into plastic tubes containing EDTA: S1: $10 \mathrm{~min}$ after catheter insertion, just before ingestion of a capsule containing either a placebo (control condition) or $32 \mathrm{mg}$ methylprednisolone (MEDROL, Upjohn); S2: $3 \mathrm{~h}$ after ingestion of a placebo or the drug. The subjects ran then for 20 min at the predetermined speed. Exercise $\mathrm{VO}_{2}$ was monitored continuously during the downhill run and the running speed was adjusted if necessary so as to keep steady state $\dot{\mathrm{VO}}_{2}$ as close as possible to the predetermined value. Two post-exercise blood samples were drawn: S3: immediately after the run; S4: after $20 \mathrm{~min}$ recovery. Three millitres of whole fresh blood were taken from each tube for leucocyte count using a SYSMEX E-5000. The remaining $7 \mathrm{ml}$ were centrifuged and the supernatants were kept in sealed tubes at $-70^{\circ} \mathrm{C}$ for further analysis. Plasma elastase concentration was assessed using an immunoactivation method (Merck, Kit 11332). ${ }^{13}$ Plasma myeloperoxidase concentration MPO was assessed according to the radioimmunological method described by Pincemail et al. ${ }^{14}$ Preliminary experiments showed that methylprednisolone was without effect on elastase and myeloperoxidase assays (unpublished results).

The occurrence of DOMS was recorded $48 \mathrm{~h}$ after downhill runs by asking the subjects whether they suffered from muscle pain during walking upstairs and downstairs. Muscle soreness perception was evaluated using an arbitrary 10-point rating scale $(1=$ no pain, $10=$ very severe $)$.

Values are expressed throughout this study as means $\pm \mathrm{SD}$. A two-way analysis of variance with repeated measurements and Bonferroni's post-boc test identified means which differed as a function of time in the two experimental conditions. Differences between mean PMN and EL values obtained after placebo and methylprednisolone at each time point were evaluated using Student's $t$-test for paired observations. Statistical significance was accepted at the 0.05 level. Given the low number of subjects, statistical analysis was used as a trend indicator.

\section{Results}

The subjects' $\mathrm{VO}_{2} \max$ averaged $55 \pm 3 \mathrm{ml}$ $\mathrm{O}_{2} / \mathrm{kg}$ per min. Mean running speed was $11 \pm 2 \mathrm{~km} / \mathrm{h}$.

The effects of methylprednisolone on absolute circulating neutrophil count (PMN) at rest and during exercise are shown in Fig. 1. While resting PMN count remained unchanged after placebo, a marked neutrophilia $(+103 \%$ of basal value; $p<0.02)$ was observed $3 \mathrm{~h}$ after methylprednisolone ingestion. At this time point, PMN count in the placebo group was significantly lower than in the methylprednisolone group $(p<0.05)$. During both exercise trials, placebo and methylprednisolone, PMN counts were increased by $46 \%$ and $19 \%$ $(p<0.05)$, respectively. Mean PMN values after methylprednisolone were significantly greater than after placebo in blood samples taken just before, immediately after exercise and during recovery $(p<0.05)$. During discovery, PMN decreased slightly but remained significantly different from baseline values measured $3 \mathrm{~h}$ before exercise.

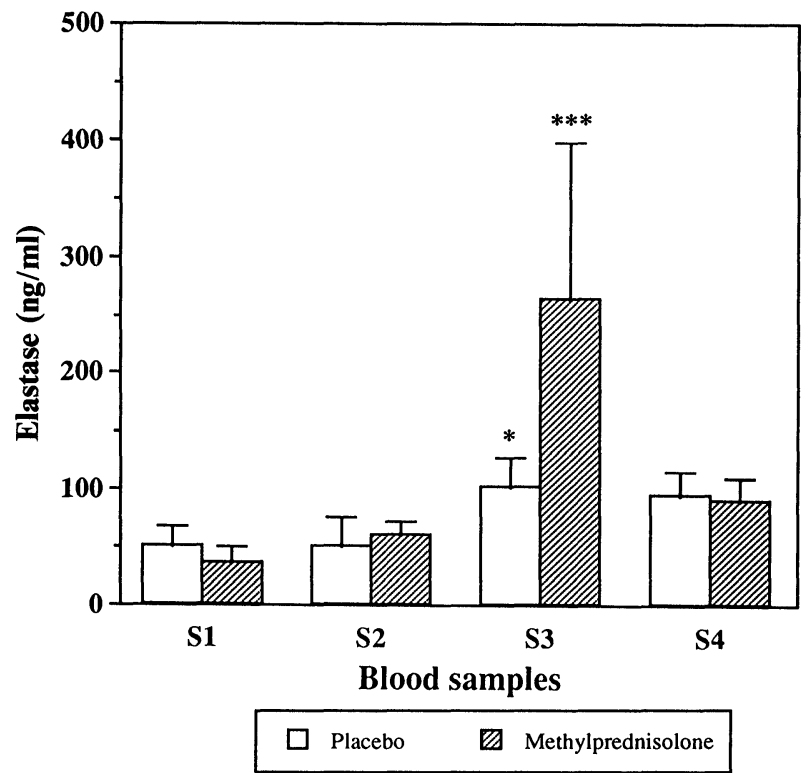

FIG. 1. Blood polymorphonuclear neutrophil counts as a function of time in the two experimental conditions (S1: $10 \mathrm{~min}$ after catheter insertion; S2: $3 \mathrm{~h}$ after ingestion of a capsule containing either a placebo or $32 \mathrm{mg}$ methylprednisolone; S3: immediately after the run; S4: after $20 \mathrm{~min}$ recovery). Vertical bars are SD $(n=0.4)$. ${ }^{*}$ Significantly different from pre-exercise level (S2): $p<0.05 ;{ }^{* *}$ Significantly different from baseline level: $p<0.02$ 
At this time point, mean EL were $51 \pm 16$ $\mathrm{ng} / \mathrm{ml}$ and $36 \pm 14 \mathrm{ng} / \mathrm{ml}$ in placebo and methylprednisolone experiments, respectively. As shown in Fig. 2, exercise was accompanied by significant changes of EL in both trials. Maximal EL values reached after $20 \mathrm{~min}$ exercise were $102 \pm 26$ and $263 \pm 134 \mathrm{ng} / \mathrm{ml}$ in control $(p<0.05)$ and methylprednisolone $(p<0.02)$ groups, respectively. Plasma elastase levels reached at the end of exercise on methylprednisolone were significantly higher than after placebo $(p<0.05)$. After $20 \mathrm{~min}$ recovery, plasma elastase levels did not differ significantly from baseline values just before exercise. There was a significant relationship between EL and PMN in methylprednisolone trial only $(r=0.72 ; p<0.005)$.

Resting MPO was unaffected by placebo and methylprednisolone absorption. The patterns of MPO changes suring exercise and recovery (Fig. 3) were similar in both experimental conditions. A significant increase $(+110 \% ; p<0.05)$ of mean MPO values was observed after $20 \mathrm{~min}$ exercise. This variable returned to pre-exercise levels on recovery. All subjects complained of muscle soreness $48 \mathrm{~h}$ after exercise. The rate of soreness ranged between 3 and 7 in the two trials.

While methylprednisolone had no effect on the exercise-induced change of MPO, this drug amplified the exercise-induced increase of EL. The positive relationship between EL and PMN suggest that this effect of methylprednisolone could be explained, at least in part, by the increase of PMN count.

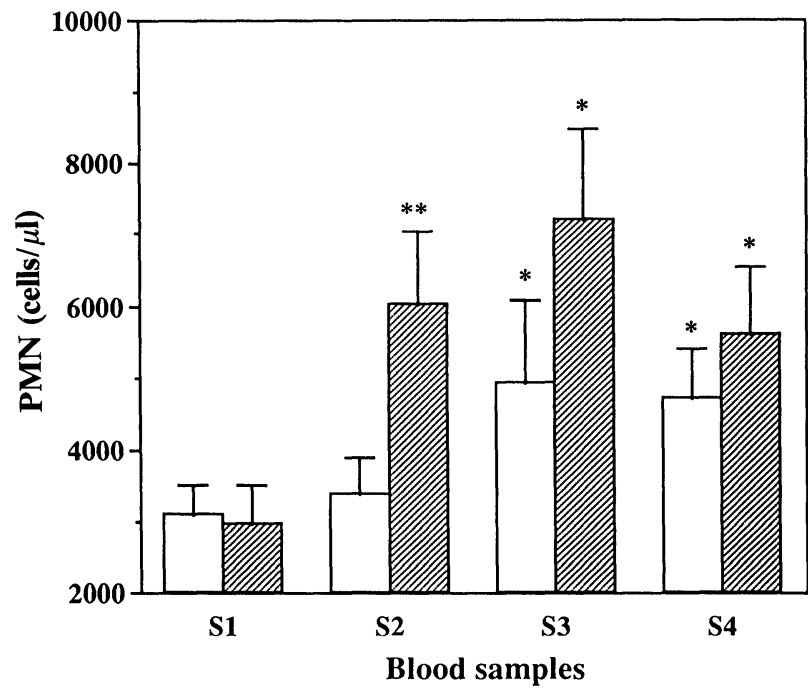

$\square$ Placebo $\bigotimes$ Methylprednisolone

FIG. 2. Mean plasma concentration of elastase as a function of time in the two experimental conditions (see Fig. 1). * " *Significantly different from baseline level: $p<0.05$ and $p<0.005$, respectively.
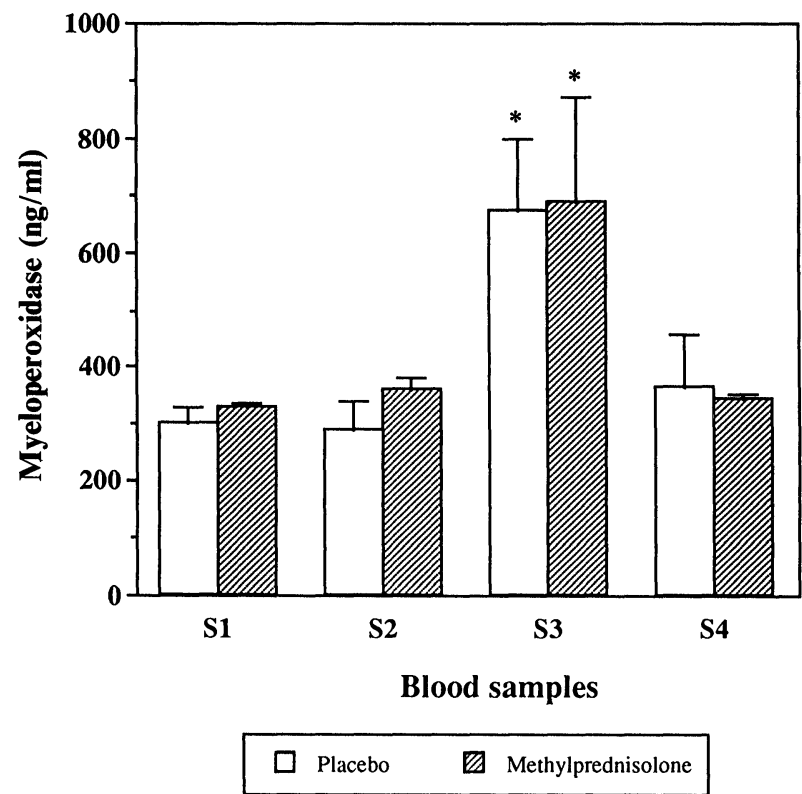

FIG. 3. Mean plasma concentration of myeloperoxidase as a function of time in the two experimental conditions (see Fig. 1). *Significantly different from baseline level: $p<0.05$

\section{Discussion}

The marked neutrophilia observed after methylprednisolone is in agreement with literature dealing with the effects of corticoids on PMN count, ${ }^{15}$ and clearly demonstrates the efficacy of drug absorption. While the influx of cells from the bone marrow was probably the principal mechanism underlying the increase in resting PMN count following methylprednisolone, ${ }^{15}$ the early neutrophilia observed during the running test probably resulted from PMN demargination under the effects of increased secretion of catecholamines and hemodynamic changes associated with exercise. ${ }^{16}$

The significant increase of EL and MPO accompanying exercise is in agreement with the results of previous studies where strenuous exercise was found to induce PMN activation. ${ }^{1-5}$ Although the mechanisms responsible for this transient activation of PMNs are not fully understood, there are, however, several lines of evidence suggesting that this early PMN response could be a part of an acute inflammatory response to muscles and/or connective tissue damage. ${ }^{3,5}$ Consistent with this view are the significant relationship between MPO and the plasma level of the complement split product $\mathrm{C} 5 \mathrm{a}$ as well as the changes of these variables following a similar time frame during a $20 \mathrm{~min}$ exercise test at $80 \% \quad \dot{V O}_{2}$ max. ${ }^{4}$ The DOMS symptoms reported in all subjects lend further support to this assumption. According to Smith, ${ }^{8}$ it seems tenable to relate DOMS to an acute inflammatory reaction where tissue injury could be the initiating event. 
The recent work by Hasson et al., ${ }^{7}$ suggested that products derived from the metabolism of arachidonic acid could be involved in this process. They showed that an anti-inflammatory drug inhibiting the enzyme cyclooxygenase (Ibuprofen) was effective in reducing DOMS and improving performance recovery when given before strenuous eccentric exercise. These findings prompted us to verify whether a steroidal anti-inflammatory agent inhibiting the metabolism of arachidonic acid could reduce DOMS and decrease the exercise-induced release of elastase and myeloperoxidase. Surprisingly, we found that the increase in plasma elastase level accompanying exercise was markedly amplified by methylprednisolone. The precise mechanisms by which methylprednisolone amplified the exercise-induced elastase release and the possible consequences of this phenomenon obviously need further studies. Nevertheless, the significant relationship between PMN and EL, suggest that this effect could be related to an influx of cells from the bone marrow. It should be kept in mind, however, that other mechanisms could also be involved, such as an alteration of the removal rate of elastase from the blood.

On the contrary, there were no effects of methylprednisolone on the exercise-induced changes of MPO. As myeloperoxidase and elastase are contained within the same granules of PMN, this lack of effect of methylprednisolone on MPO is an unexpected finding that we are unable to explain. Relevant experiments in this field would verify whether methylprednisolone could alter the metabolism of elastase and myeloperoxidase.

The subjective DOMS symptoms did not seem to be altered by the drug. It should be kept in mind, however, that only four subjects participated in this study. Furthermore, the method we used to evaluate muscle soreness was purely empirical. As a consequence, these results are at best suggestive. Further experiments with more appropriate methods of DOMS scoring may prove helpful in studying the effects of anti-inflammatory drugs on muscle soreness.
Despite the low number of subjects who took part in this study, these preliminary results showed that a single oral dose of methylprednisolone did not decrease the exercise-induced release of two specific markers of PMN activation. In comparison with placebo, methylprednisolone seemed to amplify the exercise-induced increase of EL.

\section{References}

1. Schaefer RM, Kokot K, Heidland A, Plass R. Jogger's leukocytes. $N$ Engl $J$ Med 1987; 316: 223-224.

2. Pincemail J, Camus G, Roesgen A, et al. Exercise induces pentane production and neutrophil activation in humans. Effects of propranolol. Eur J Appl Pbysiol 1990; 61: 319-322.

3. Camus G, Pincemail J, Lamy M. Is there an analogy between sepsis and strenuous physical exercise in the process of neutrophil activation in man a working hypothesis. In: Vincent JL, Ed., Update in Intensive Care and Emergency Medicine. Berlin, Heidelberg: Springer Verlag, 1991: 206-212.

4. Camus G, Pincemail J, Duchateau J, et al. Complement and polymorphonuclear neutrophil activation during submaximal exercise in man. Arch In Physiol Biochem 1991; 100: P14.

5. Camus G, Pincemail J, Ledent M, et al. Plasma levels of polymorphonuclear elastase and myeloperoxidase after uphill walking and downhill running at similar energy cost. Int J Sports Med 1992; 13: 443-446.

6. Herbaczynska-Cedro K, Staszewska-Barczak J, Janczewska H. Muscula work and the release of prostaglandin-like substances. Cardiovasc Res 1976; 10: 413-420.

7. Hasson SM, Daniels JC, Divine JG, et al. Effect of ibuprofen use on muscle soreness, damage, and performance: a preliminary investigation. Med Sci Sports Exerc 1993; 25: 9-17.

8. Smith LL. Acute inflammation: the underlying mechanism in delayed onset muscle soreness? Med Sci Sports Exerc 1991; 23: 542-551.

9. Flower RJ. Lipocortin and the mechanism of action of the glucocorticoids. Br J Pharmacol 1988; 94: 987-1015.

10. Friden J, Sfakianos PN, Hargens AR. Muscle soreness and intramuscular fluid pressure: comparison between eccentric and concentric load. $J$ Appl Physiol 1986; 61: 2175-2179.

11. Camus G, Thys H. An evaluation of the maximal anaerobic capacity in man. Int J Sports Med 1991; 4: 349-355.

12. Margaria R, Cerretelli P, Aghemo P, Sassi G. Energy cost of running J Appl Physiol 1963; 18: 367-370.

13. Dreher R. Mechanized immunoactivation test for the measurement of human granulocyte elastase. In: Lang H, Fritz H, Redl H, eds. Elastase Expert Meeting. Vienna: 1988: 28-33.

14. Pincemail J, Deby-Dupont G, Deby C, et al. Fast double antibody radioimmunoassay of human granulocyte myeloperoxidase and its application to human plasma. J Immunol Meth 1991; 137: 181-191.

15. Dale DC, Fauci AS, Guerry DP, Wolff SM. Comparison of agent producing a neutrophilic leukocytosis in man. J Clin Invest 1975; 56: 808-813.

16. McCarthy DA, Dale MM. The leucocytosis of exercise. A review and model. Sports Med 1988; 6: 333-363.

Received 28 May 1993;

accepted in revised form 8 June 1993 


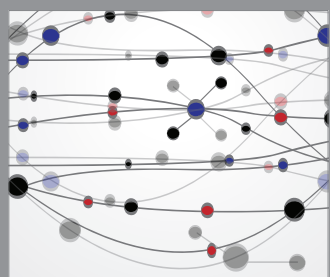

The Scientific World Journal
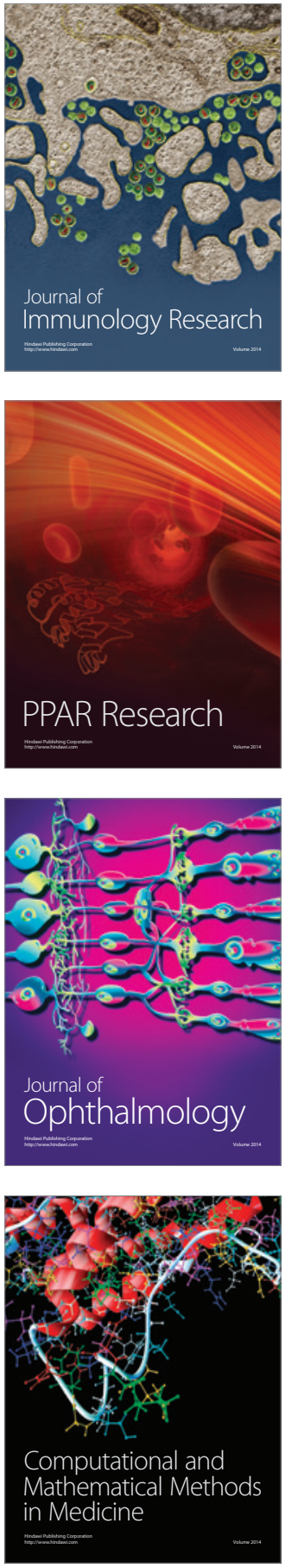

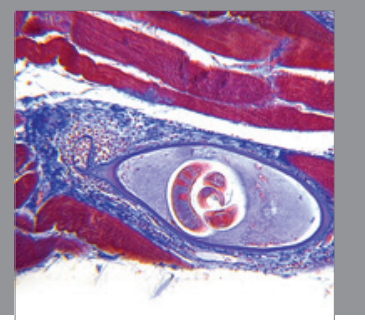

Gastroenterology

Research and Practice
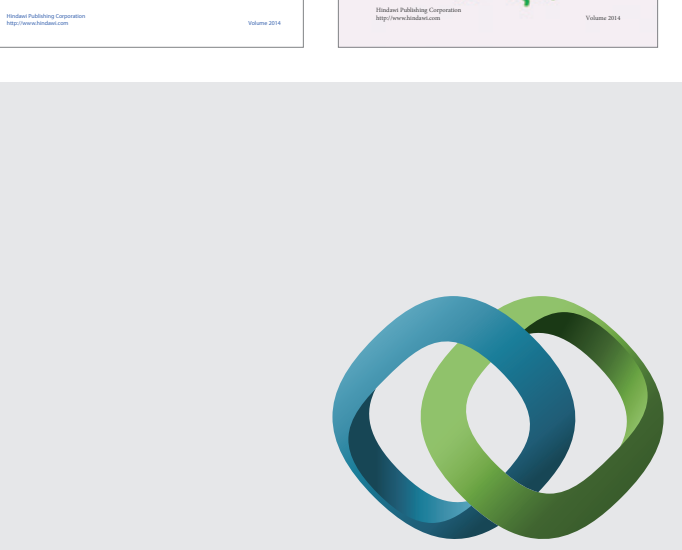

\section{Hindawi}

Submit your manuscripts at

http://www.hindawi.com
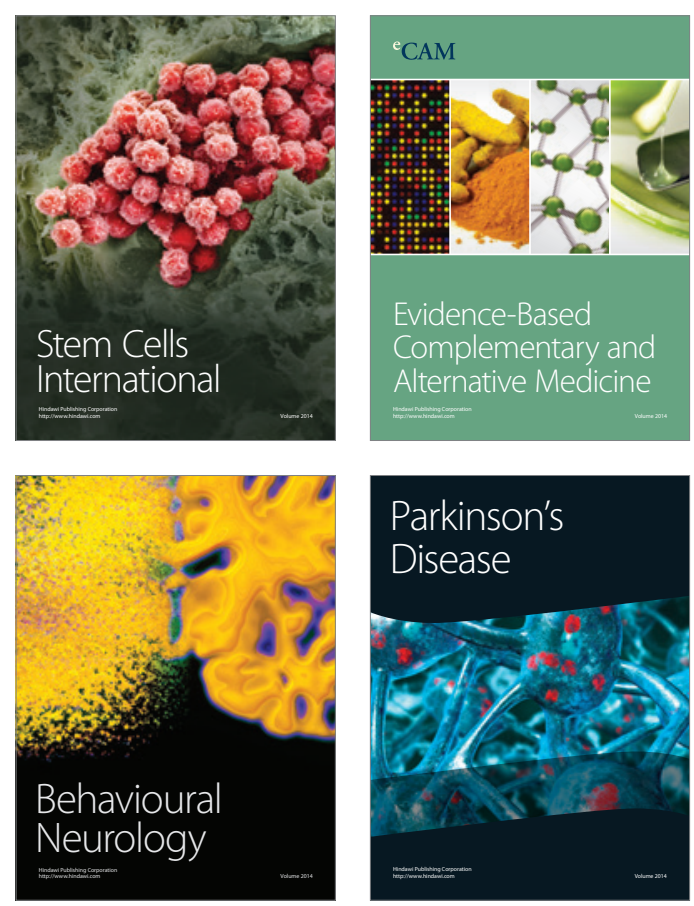

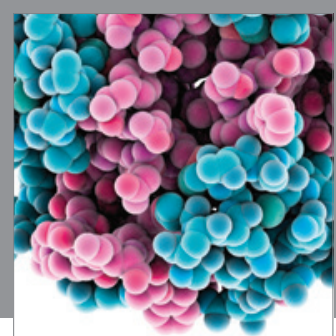

Journal of
Diabetes Research

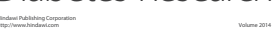

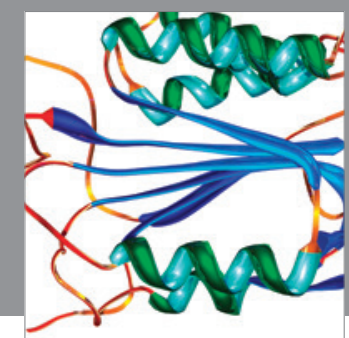

Disease Markers
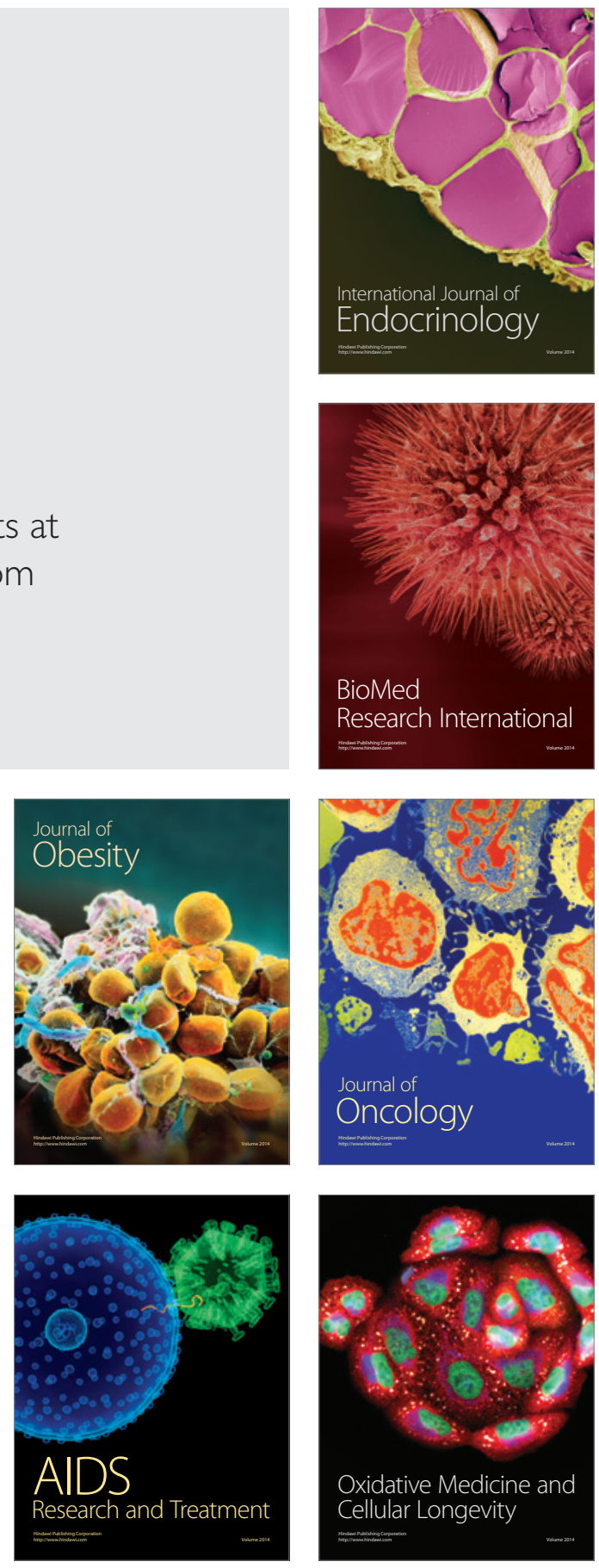